\title{
General Framework for Real-time Implementation of Balancing Services Market Outcome ${ }^{\star}$
}

\author{
A. Virag, ${ }^{*}$ A. Jokić, ${ }^{*}$ P.P.J. van den Bosch * \\ * Electrical Engineering, Eindhoven University of Technology, 5612 AZ Eindhoven, \\ The Netherlands (e-mail: a.virag, a.jokic, p.p.j.v.d.bosch@tue.nl).
}

\begin{abstract}
Load frequency control (LFC) is widely used for real-time balancing connected power systems. With the deregulation of power system markets, there is a necessity for adapting the current LFC to support market-based operation. In this paper we propose a general balancing tool which covers both, regulated, and deregulated load frequency control. The proposed approach enables implementation of conventional, two-sided markets possible as well as implementation of the integrated markets for balancing reserves, i.e. cooperation in provision of reserves among different control areas.
\end{abstract}

Keywords: Load-frequency control, power systems control, ancillary services, ancillary service market, balancing reserves.

\section{INTRODUCTION}

Load frequency control (LFC) is a well-known method for balancing interconnected power systems. It maintains the frequency of each control area ${ }^{1}$ and power exchanges among the areas at their reference values.

Even though the LFC is a commonplace in power system balancing, in practice the implementation varies from one control area to another, see e.g. Tractebel [2009]. There is a remarkable variety in its design and activation with no unified, generally accepted implementation. Furthermore, the current implementations in most control areas do not consider distribution of balancing reserves within the transmission network which has limited power transfer capacity. Therefore, these methods do not guarantee that LFC actions will not jeopardize system's operation by driving it to an unfeasible state. LFC attracted a lot of research attention and has been extensively studied in literature; see an overview paper of Kumar and Kothari [2005]. The research interest spanned various control techniques and differed from optimal control to adaptive and self-tuning LFC schemes. With the deregulation of power system markets, new research challenges, e.g. proper market design and implementation, came into focus. Some of the first works on the implementation of bilateral contracts for power among different control-areas in real-time are Kumar et al. [1997] and Donde et al. [2001]. Their work has later been extended to explore the feasibility of the implementation of bilateral contracts for balancing reserves in Nobile et al. [2001] and Abraham et al. [2009].

Recently, new ideas about organization and operation of power markets and power systems have been presented, see Jokić [2007], $\S 4$, or van den Bosch et al. [2010]. The mainline of these ideas is changing the operational structure of currently

\footnotetext{
* This work is supported by the European Commission Research Project FP7-ICT-249096 Price-based Control of Electrical Power Systems (E-Price), www.e-price-project.eu.

1 A control area is an area under supervision and responsibility of an independent system operator (ISO) in North America or transmission system operator (TSO) in Europe. In the rest of this work, we use TSO.
}

implemented single-buyer markets for balancing reserves to two-sided markets for balancing reserves. However, although it is accepted that introducing such markets would increase the efficiency of the system, the possibilities of the implementation in real time have not yet been fully explored.

In this paper, we present a general proposal for implementing real-time power balancing. The proposed implementation encompasses regulated and deregulated power system operation, cooperation among different control areas or new operational ideas, as explained later. The main idea is implementing the market outcome by creating a balancing participation mapping.

\section{NOTIONS AND DEFINITIONS}

Consider a connected undirected graph as an abstraction of transmission network of a power system. We associate the nodes with the buses in the transmission network and the edges with the power lines of the network. We use $\mathcal{V}:=\{k \mid k=$ $1, \ldots, N\}$ to denote the set of nodes. Suppose that the network consists of a set of control areas denoted by $\mathcal{Z}=\left\{\mathcal{Z}_{1}, \ldots \mathcal{Z}_{K}\right\}$. In the remainder we assume that the enumeration of the nodes is such that the first $n_{1}$ nodes belong to the first control area, the next $n_{2}$ nodes $\left\{n_{1}+1, \ldots, n_{1}+n_{2}\right\}$ define the second control area, etc.

With each node $k \in \mathcal{V}$ we associate variables $\Delta p^{k} \in \mathbb{R}$ and $\Delta q^{k} \in \mathbb{R}$. These values denote the real-time power deviations from scheduled (e.g. via contracts made in forward time energy market) controllable and predicted uncontrollable injections at node $k$, respectively. Positive values correspond to a flow of power into the network (production), while negative values denote power extracted from the network (consumption) at that particular node. We use the abbreviations

$$
\begin{aligned}
\Delta p & :=\operatorname{col}\left(\Delta p^{1}, \ldots, \Delta p^{N}\right), \\
\Delta q & :=\operatorname{col}\left(\Delta q^{1}, \ldots, \Delta q^{N}\right) .
\end{aligned}
$$

We refer to $\Delta q$ as to a vector of the uncontrollable power deviations or the uncertainty. 


\section{POWER SYSTEM OPERATION}

\subsection{Physics of the power system}

It is convenient to represent the whole power system as a connection of control areas, one of which is illustrated in Fig. 1. Fig. 1 without the part highlighted in red, represents a classical, already implemented power balancing control. Blocks $G^{i}(s)$

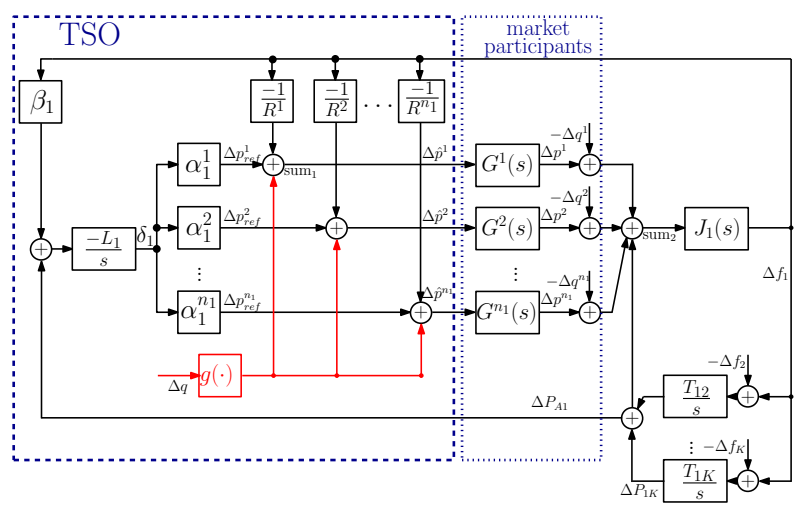

Fig. 1. Representation of a power system control area with market outcome implementation (depicted in red).

represent power generators. They typically contain a second or third order linear transfer function, depending on the type of the generator, see Kundur [1994]. The gain of each generator block is equal to 1 in steady-state. Therefore, in steady state we have that $\Delta \hat{p}^{1}=\Delta p^{1}$. Positive parameter $\beta_{i}$ denotes the composite frequency response characteristic, see Kundur [1994]. $J_{i}(s)=$ ${ }^{1} M_{i} s+D_{i}$ models the inertia of the control area, with $D_{i}$ a damping constant, and $M_{i}$ equivalent inertia of the connected system. $T_{i j} \in$ is a tie-line reactance between control area $Z_{i}$ and its neighboring area $Z_{j}$. If control areas $Z_{i}$ and $Z_{j}$ are not neighbors, $T_{i j}=0 .{ }_{R^{k}}^{1}$ is a proportional frequency controller with $R^{k}$ being referred to as droop or speed regulation. $\alpha_{i}^{k}$ is a parameter that determines the participation of generator $G_{i}(s)$ in compensation of the area control error. Factor $L_{i}$ denotes the controller gain of the system. $\Delta f_{i}$ and $\Delta P_{A i}$ are deviation from the reference value of real-time frequency and deviation of the aggregated scheduled power exchange with the neighboring control areas, respectively. $\delta_{i}$ is a control signal that should be distributed to all generators in a control area to bring about balance in the system. The coefficient $\alpha_{i}^{k}$ is a LFC participation factor of a generator $\mathrm{k}$ in a control area $Z_{i}$.

Remark 3.1. In the current power system operation practice, numerical values of the participation factors $\alpha_{i}^{k}$ are determined based on bids for balancing reserves. These coefficients therefore represent the link between markets/economy and real-time control of the physical power system.

In the described system, variables that are easily measurable and accessible are $\Delta f_{i}$ and $\Delta P_{A i}$. At the moment, they are used to balance the system, through the feedback. Primary control is represented by proportional control action. In Section 5, we propose a modified way of balancing the system, based on the assumption that real-time measurement of $\Delta q$ is available. This assumption could in theory be achieved, see Remark 5.7 and 5.8 .

\subsection{Economics behind the power system}

In the regulated environment all the blocks depicted in Fig. 1 are under the regulation of one entity which has nominally full knowledge about the system. With the deregulation, the role of different entities has to be clearly defined. Thus, the balancing of a control area is a responsibility of a TSO, see dashed block in Fig. 1, while power generators are grouped under several power companies which we refer to as market participants, see dotted block in Fig. 1, whose goal is to make profit by producing and trading power. Market participants' responsibility is respecting the market outcomes while taking into account their own constraints and uncertainty.

A TSO is obliged to balance the system in real time by activating balancing reserves according to the balancing market outcome. Therefore, its duty is (i) real-time balancing of the system by (ii) implementing market outcome while taking into account constraints of the grid. It is essential that the implementation of the market outcome does not cause congestion in the system during the real-time balancing.

\subsection{Power system behavior in a steady-state}

In this subsection, we introduce the balancing participation mapping $g(\Delta q)$. We give it an interpretation of a mapping which defines, based on the market outcome, which $\Delta p$ has to be activated as a response to the real-time uncertainty $\Delta q$, i.e., $\Delta p=g(\Delta q)$. This mapping has a two-folded purpose $(i)$ real-time balancing of the system by (ii) implementing market outcome while taking into account constraints of the grid. In other words, mapping $g(\Delta q)$ mathematically formalizes the role of a TSO in steady-state. Task (ii) is related to both, economics and physics, whereas task $(i)$ concerns only physics of the power system.

The relation $\Delta p=g(\Delta q)$ characterizes the closed-loop behavior of the power system in steady-state. The function $g(\cdot)$ necessarily has to satisfy the global power balance relation, i.e. it has to hold that $\sum_{k=1}^{N} \Delta p^{k}=\sum_{k=1}^{N} g^{k}(\Delta q)=\sum_{k=1}^{N} \Delta q^{k}$, where $g^{k}(\Delta q)$ is the market base reference power adjustment of a generator $k$. Since the mapping $g(\cdot)$ describes the mechanism of how power imbalances in the system will be compensated for, its proper formulation enables us to account for transmission system constraints during the ancillary services market clearing, e.g. before (in forward time) the real-time operation.

Remark 3.2. Similarly as in Remark 3.1, the mapping $g(\cdot)$ is to be considered as a link between balancing reserves markets/economy forward-time system operation and control of the physical system real-time operation.

Example 3.3. Currently, the physical system constraints are taken into account before the real-time operation and after receiving offers for balancing, see e.g. TenneT [2011]. This task is sometimes called congestion management. A TSO discards some bids if their activation could cause congestion in the system. The implementation of the market outcome with respect to constraints can be mathematically formalized as creating the balancing participation mapping $g(\cdot)$.

There are many ways to create $g(\Delta q)$. One of them is as described in the example above, other are optimal dispatch, or robustly optimal distribution of balancing reserves in the grid. In this paper, we consider $g(\Delta q)$ to be given. The goal is to find a real-time controller, which balances the power system 
by implementing the given balancing reserve market outcome, $g(\Delta q)$.

\section{PROBLEM FORMULATION}

The real-time power balancing problem, as considered in this paper, is formalized as follows.

Problem 4.1. Given a function $g(\Delta q)$, which represents the forward-time market outcome, design a real-time controller such that the following objective is met for any constantvalued exogenous uncontrollable real-time disturbance $\Delta q$ : the state of the closed-loop power system globally converges to an equilibrium point which is characterized with the relation $\Delta p=g(\Delta q)$.

In connection with Problem 4.1, we define the following subproblems:

(i) ensuring existence of an equilibrium point described by $\Delta p=g(\Delta q)$;

(ii) ensuring stability of the equilibrium point $\Delta p=g(\Delta q)$.

We consider the problem $(i)$ in the following section.

\section{IMPLEMENTATION OF THE BALANCING PARTICIPATION MAPPING}

In this section we show several implementations of the market outcome (e.g. of the function $g(\cdot)$ ) in real-time. Firstly, we show that the system depicted in Fig. 1 is an implementation of $g(\Delta q)$. Note again that Fig. 1 represents a classical, already implemented power balancing control extended by a block highlighted in red, which contains a balancing mapping $g(\Delta q)$. The output of that block is fed to power generators as a reference input.

Assumption 5.1. Suppose that we have an estimation of $\Delta q$, which is exact at least for a case when the system is in steady state. Furthermore, we assume that the signal $\Delta q$ is a constantvalued exogenous uncontrollable real-time disturbance, i.e. a quasi static signal.

Proposition 5.2. If the system in Fig. 1 is stable, under assumption Asmp. 5.1, the relation $\Delta p=g(\Delta q)$ is imposed in steady state.

Proof. In steady-state, all inputs to the integrators are zero, and hence: $\beta_{i} \Delta f_{i}+\Delta P_{A i}=0, \forall i$ and $\Delta f_{i}=\Delta f_{j}, \forall i, j$, see Fig. 1. As proven in Appendix, from these equations follows that $\Delta f_{i}=0, \forall i, \Delta P_{A i}=0, \forall i$. Due to $\Delta f_{i}=0$ and block $J_{i}(s)$ acting as a nonzero gain in steady-state, input to the block $J_{i}(s)$ is necessarily zero. Then, from the sum element in front of the block $J_{i}(s) \operatorname{sum}_{2}$ in Fig. 1, it holds $\sum_{k=1}^{n_{1}} \Delta p^{k}-$ $\sum_{k}^{n_{1}} g^{k}(\Delta q)-\Delta P_{A 1}=0$, or

$$
\sum_{k}^{n_{1}} \Delta p^{k}=\sum_{k}^{n_{1}} g^{k}(\Delta q) .
$$

From the element sum 1 in Fig. 1, it holds $\alpha_{i}^{k} \delta_{i}-{ }_{R^{k}}^{1} \Delta f_{i}+$ $g^{k}(\Delta q)=\Delta p^{k}, \forall k$. If the latter equation is summed over all generators in the control area, and by taking into account that $\Delta f_{i}=0$ in steady-state, it holds

$$
\sum_{k}^{n_{1}} \Delta p^{k}=\sum_{k}^{n_{1}} \alpha_{i}^{k} \delta_{i}+\sum_{k}^{n_{1}} g^{k}(\Delta q) \text {. }
$$

From both, (2) and (3), it follows $\sum_{k}^{n_{1}} \alpha_{i}^{k} \delta_{i}=0$. Therefore, Fig. 1 implements any market outcome represented by mapping $g(\Delta q)$.
Remark 5.3. $\sum_{k}^{n_{1}} \alpha_{i}^{k} \delta_{i}=0$ implies $\delta_{i} \sum_{k}^{n_{1}} \alpha_{i}^{k}=\delta_{i} \times 1=0$, i.e. $\delta_{i}=0$. The steady state is independent on the choice of $\alpha_{i}^{k}$. Assumption 5.4. Assume the mapping $g(\Delta q)$ is a given linear function of $\Delta q$, i.e., $g(\Delta q)=\boldsymbol{\Lambda} \Delta q$, where $\boldsymbol{\Lambda}$ is a $N \times N$ matrix created as a result of market outcome. We will call the matrix $\Lambda$ participation matrix. To ensure global power balance for an arbitrary $\Delta q$, the matrix $\boldsymbol{\Lambda}$ necessarily has to satisfy the following condition

$$
\mathbf{1}_{N}^{\top} \boldsymbol{\Lambda}=\mathbf{1}_{N}^{\top}
$$

where $\mathbf{1}_{N}$ is the $N$-dimensional column vector with all entries equal to one. In other words, all the columns of the participation matrix $\Lambda$ have to sum up to one.

The condition (3) implies the overall system power balance since $\sum_{k=1}^{N} \Delta p^{k}=\mathbf{1}_{N}^{\top} \Delta p=\mathbf{1}_{N}^{\top} \boldsymbol{\Lambda} \Delta q=\mathbf{1}_{N}^{\top} \Delta q$.

Remark 5.5. Since the mapping $g(\Delta q)$ represents the market outcome and is chosen so that all the columns sum to 1 , it holds $\sum_{k}^{n_{1}} g^{k}(\Delta q)=\sum_{k}^{n_{1}} \Delta q^{k}$. Therefore, equation (2) represents the balancing equation. Note that the condition (4) imposed on the function $g(\cdot)$ is a necessity for consistency with (2).

Remark 5.6. In case the equilibrium point is defined by a linear mapping, e.g. $g(\Delta q)=\boldsymbol{\Lambda} \Delta q$, since the rest of the system is linear, the stability analysis is straightforward and omitted in the paper for the sake of brevity. However, for a general case, proving stability, i.e. solving the subproblem (ii) is a difficult task.

Remark 5.7. Asmp. 5.1 is not realistic, since having the perfect estimation of $\Delta q$ means knowing at every time instance every change in the system. However, in case all power line flows in the system $\Delta P_{i j}$ and all generator injections $\Delta p^{k}$ are known, it is possible to estimate $\Delta q^{k}$. The equilibrium equation for every node is: $\Delta p^{k}-\Delta q^{k}+\sum_{j \in \mathcal{N}_{i}} \Delta P_{i j}=0$, where $j \in \mathcal{N}_{i}$ are all neighboring nodes of a node $i$.

Remark 5.8. If Assumption 5.1 does not hold, i.e. if there is an estimation error, equation (2) is still valid. However, $\sum_{k=1}^{n_{1}} \alpha_{i}^{k} \delta_{i} \neq 0$. Frequency and tie-line deviations are partly compensated through function $g(\cdot)$, and partly by contribution of the feedback loop through the choice of coefficients $\alpha_{i}^{k}$. Depending on accuracy of estimation, contribution $\sum_{k}^{n_{1}} \alpha_{i}^{k} \delta_{i}$ is arbitrary small. This considerably relaxes Assumption 5.1.

The above described implementation only allows for existence of two-sided balancing reserve markets, see Remark 5.3. To

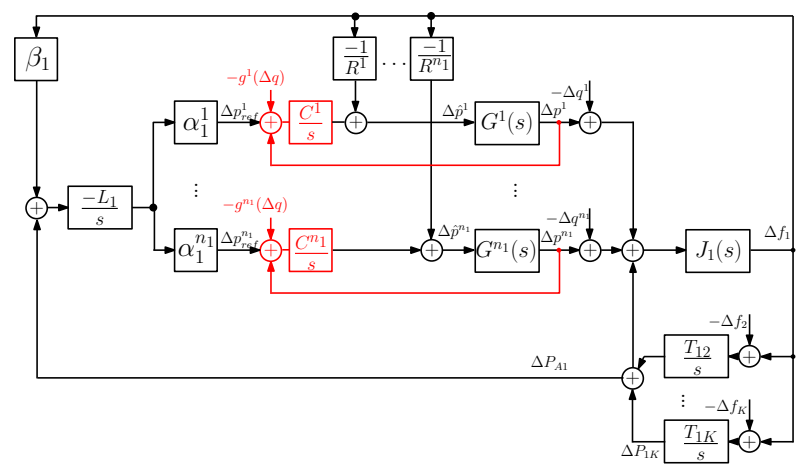

Fig. 2. Implementation of market outcome by a local integrator.

relax this assumption, another possible implementation of a market outcome is illustrated in Fig. 2. In this case, each generator has an additional integrator, which integrated the difference 
of the generator power output and sum of market outcome and ACE participation. This implementation is presented in Abraham et al. [2009]. The proposed I controller can be changed into PI-controller for faster step response. However, stability analysis of such a system is challenging. In this case, it is possible for a generator to participate in two-sided markets for reserves and have a contract with TSO for provision of emergency power.

\section{FROM REAL-TIME CONTROL TO BALANCING PARTICIPATION MAPPING}

In the previous section we have shown how to implement arbitrary $g(\cdot)$. In this section we are concerned with the inverse problem. More precisely, we start with the given real-time control structure and determine the corresponding participation matrix $\boldsymbol{\Lambda}$. This is done for regulated reserve activation, currently applied secondary control, and future two-sided markets. Furthermore, we determined the structure of the participation matrix $\Lambda$ in case of cooperation in provision of balancing reserves among control areas.

The purpose of this section is therefore to relate the considered real-time control schemes with the notion of participation mapping, which serves an important link between forward time and real-time operation of the system, see Remarks 3.1 and 3.2.

\subsection{Classical load frequency control implementation}

Here, we show that in case of LFC control before the deregulation, mapping $\Delta p=g(\Delta q)$ simplifies to a linear mapping of a specific structure described by $\Delta p=\Lambda \Delta q$, where $\Lambda$ is a balancing participation matrix.

Proposition 6.1. Consider a system which consists of $K$ control areas with $n_{i}$ denoting a number of generators in an area $Z_{i}$. The participation mapping for the standard LFC is then given by $\Delta p=\boldsymbol{\Lambda} \Delta q$, where $\boldsymbol{\Lambda}=\operatorname{diag}\left(\boldsymbol{\Lambda}_{1}, \ldots, \boldsymbol{\Lambda}_{K}\right)$, and $\boldsymbol{\Lambda}_{i}=\alpha_{i}^{k} 1_{n_{i}}^{\top}$, with $\alpha_{i}^{k} \in \mathbb{R}^{n_{i}}$ vector of participation factors in a control area $Z_{i}$.

Proof. In steady-state, all the inputs in the integrators in Fig. 1 are zero. Therefore, as shown in the Appendix, $\Delta f_{i}=0$, $\Delta P_{A i}=0$ in every control area. From Fig. 1, keeping in mind that the red line does not exist, we read the steady-state relation $\sum_{k=1}^{n_{1}} \alpha_{i}^{k} \Delta \tilde{p}=\sum_{k=1}^{n_{1}} \alpha_{i}^{k} \Delta p=\sum_{k=1}^{n_{1}} \Delta q$. Therefore, the power generators change the output in the same manner, independently on the shape of $\Delta q$ signal, i.e. the position of the imbalance in the power system,. If a vector $\alpha_{i}^{k}$ is defined as a vector of participation factors in a control area $Z_{i}$, realtime uncertainty $\Delta q$ in a control area $Z_{i}$ is distributed according to $\Delta p=\Lambda_{i} \Delta q$. The participation matrix of the overal power system is obtained by stacking each of submatrices $\boldsymbol{\Lambda}_{i}$, which represent market outcome in control area $Z_{i}$, and writing it in matrix form $\boldsymbol{\Lambda}=\operatorname{diag}\left(\boldsymbol{\Lambda}_{1}, \ldots, \boldsymbol{\Lambda}_{K}\right)$. The coefficients $\alpha_{i}^{k}$ are independent on the place of the disturbance $\Delta q$. Therefore, each submatrix $\boldsymbol{\Lambda}_{i}$ contains $n_{i}$ repeated equivalent columns. Rank of participation matrix $\boldsymbol{\Lambda}$ is equal to number of control areas in the considered interconnected power system.

\subsection{Implementation of load-frequency control after regulation}

Mapping $g(\Delta q)$ is defined as the balancing reserves market outcome before the real-time operation. After market clearing and the security analysis, the so-called $\mathrm{N}-1$ analysis, there is a set of offers whose activation is safe from the point of view of system reliability, while others are discarded, as explained in Ex. 3.3. These offers form $g(\Delta q)$ as a set of different mappings, where the domain of each mapping depends on the amount of the uncertainty in the system $\Delta q$, but does not depend on the position of the uncertainty, as the transmission constraints do not play a role any more.

Depending on the size of $\Delta q$, certain bids are activated, i.e. each node $i$ is assigned a certain participation factor $\alpha_{i}^{k}$ which defines the balancing contribution of that particular node for that particular system state. Therefore, the mapping $\Delta p=$ $g(\Delta q)$ is state dependant and can be represented by a set of different matrices that are active depending on the amount of uncertainty in the system. This makes LFC controller a gain scheduling controller.

To conclude, in this case, $\boldsymbol{\Lambda}$ is a square block diagonal matrix whose elements change depending on the state of the system according to the market outcome.

\subsection{Two-sided markets}

Introducing energy markets in power systems increased its economic efficiency Stoft [2002]. Nevertheless, nowadays only energy is merchandized on conventional, two-sided markets, while balancing reserves are traded on a single-buyer markets with the system operator (TSO) being the only buyer. The demand for balancing reserves is determined in real-time and depends on the amount of uncertainty in the system, as discussed in the previous subsection. It is reasonable to assume that introducing two-sided markets for balancing reserves, if implementable and feasible, would further improve the efficiency of power systems. We define two-sided markets for balancing reserves as markets where participants sell and, unlike in the current arrangements in power systems, also buy balancing reserves, see e.g. Jokić [2007], van den Bosch et al. [2010]. The crucial difference between the two is in determining the demand for balancing reserves. Unlike in deregulated arrangements where the demand is determined in real-time, on two-sided balancing services markets, the demand is fixed and determined before operation through market clearing. By introducing and implementing two-sided markets for balancing reserves, the involvement of the TSO would decrease, whereas the level of reliability would increase or remain the same. As shown in Sect. 5, any market outcome can be implemented as in Fig. 1. In case of two-sided markets, each submatrix has a free structure. It contains information about which generator compensates for whose imbalance and to what extent. Note that in such a way, the transmission constraints and placement of generators in the grid are taken into account. The matter of obtaining the balancing market outcome is outside the scope of this paper.

In this case, $\boldsymbol{\Lambda}$ is a square block diagonal matrix, with submatrices whose elements are sum to 1 and are freely chosen as a result of market outcome. In case of existence of transmission constraints, coefficients $\alpha_{i}^{k}$ are dependant on the position of $\Delta q$ in the grid.

\subsection{Cooperation among control areas}

As shown above, in case of classical LFC implementation, the participation matrix $\boldsymbol{\Lambda}$ has a block diagonal structure. Because of the block-diagonal structure, every control area compensates 
for its own uncertainties. This causes all the inter-area tieline flows to go to zero. Relaxing this structure introduces more degrees of freedom in the system. Furthermore, it makes implementation of cooperation among control areas possible. The cooperation is beneficial from economic point of view, but also from a reliability viewpoint.

In case of cooperation, the matrix $\boldsymbol{\Lambda}$ loses the block diagonal structure because the off-diagonal blocks are filled with participation factors of generators from other control areas. Other changes, such as changing the reference value of inter control area tie-lines, are needed.

\section{SIMULATION RESULTS}

In this section, we present several case studies to show the validity and efficiency of the proposed concept and its properties. In simulations, we assumed the grid topology as in Fig. 3,

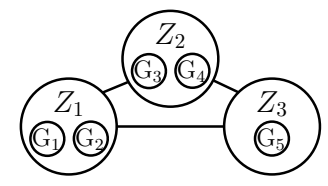

Fig. 3. Considered topology in the simulation cases.

with three control areas and five nodes in total. There is a generator in each node. The market outcome can be presented, as explained in Section 5 , by a $5 \times 5$ matrix.

\subsection{The regulated balancing}

To validate the concept, we give an example of the regulated balancing. Suppose that generator participation factors are given by

$$
\boldsymbol{\Lambda}=\left[\begin{array}{ccccc}
0.4 & 0.4 & 0 & 0 & 0 \\
0.6 & 0.6 & 0 & 0 & 0 \\
0 & 0 & 0.8 & 0.8 & 0 \\
0 & 0 & 0.2 & 0.2 & 0 \\
0 & 0 & 0 & 0 & 1
\end{array}\right]
$$

Note that the structure of $\Lambda$ indeed corresponds to the philosophy of balancing in the regulated system. Simulated uncertainty is depicted in the upper subplot of Fig. 4. At first, it acts in the node 1 in the amount -0.1 [pu MW]. At time instance $\mathrm{t}=50$ [s], the uncertainty in node 2 in the amount 0.1 [pu MW] becomes active. Both nodes are in the same control area (CA1 in Fig. 3) and after $\mathrm{t}=50[\mathrm{~s}]$, from the control area point of view, there is no uncertainty to be compensated, the generator response is expected to disappear. At $\mathrm{t}=100$ [s] At $\mathrm{t}=150$ [s] Therefore, in the first $50[\mathrm{~s}] \Delta p=[-0.04,-0.06,0,0,0]^{\top}$, between 50 [s] and $100[\mathrm{~s}] \Delta p=[0,0,0,0,0]^{\top}$, between $100[\mathrm{~s}]$ and $150[\mathrm{~s}] \Delta p=[0,0,-0.02,-0.08,0]^{\top}$ and between $150[\mathrm{~s}]$ and $200[\mathrm{~s}] \Delta p=[0,0,-0.02,-0.08,0.1]^{\top}$. The expected behavior is visible in Fig. 4.

\subsection{Cooperation among control areas}

The possibilities for balancing market coupling are studied in this example. The market outcome matrix is given as follows

$$
\boldsymbol{\Lambda}=\left[\begin{array}{ccccc}
0 & 0 & 0.7 & 0 & 0 \\
0 & 1 & 0 & 0 & 0.1 \\
0 & 0 & 0.1 & 0.1 & 0.6 \\
0.8 & 0 & 0.2 & 0.9 & 0.3 \\
0.2 & 0 & 0 & 0 & 0
\end{array}\right]
$$
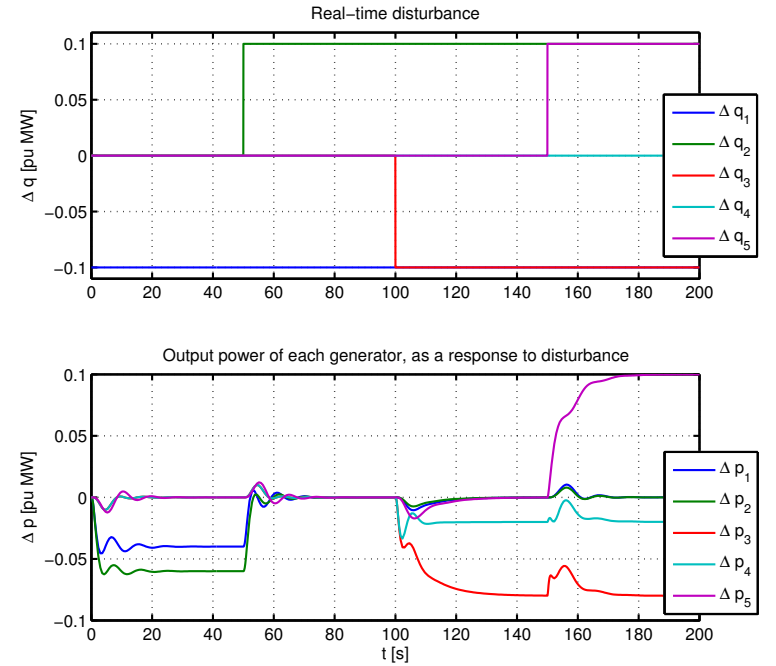

Fig. 4. The imbalance and corresponding generator response.
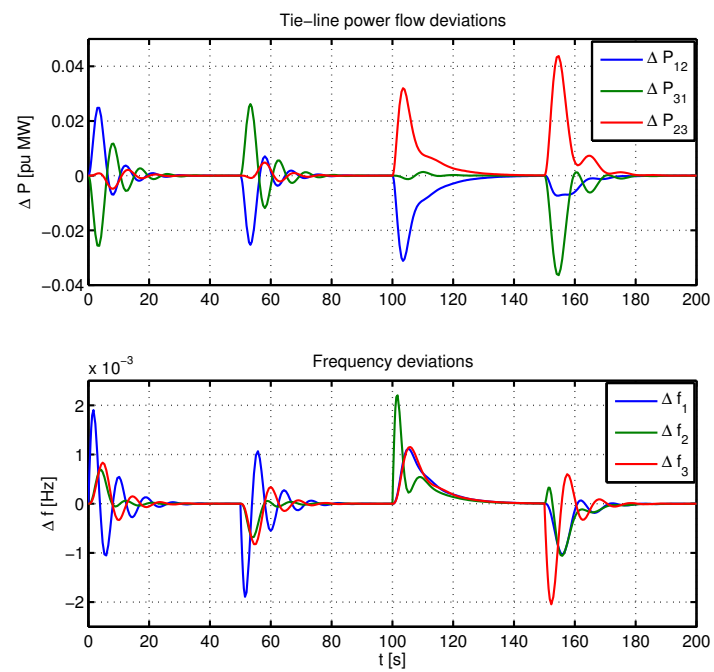

Fig. 5. The frequency and tie-line deviations.

According to given $\boldsymbol{\Lambda}$, there is cooperation among control areas in reserves provision and there is a two-sided market in each control area. The uncertainty $\Delta q$ is illustrated in the top subplot of Fig. 6. From given $\Lambda$ and $\Delta q$, the expected market outcome is $\Delta p=\Lambda \Delta q=[0,0,0,-0.08,-0.02]^{\top}$ for the first $50[\mathrm{~s}]$, and $\Delta p=[0, .01,0.06,-0.05,-0.02]^{\top}$ afterwards. The implementation of the market outcome is shown in Fig. 6.

Note that in Fig. 7, due to cooperation among control areas in reserve provision, the inter-area tie-line power flow deviations do not go to zero as it is the case in the classical LFC.

\section{CONCLUSIONS}

In this paper, we analyzed and expanded the functionality of load frequency control for future challenges. Prior work of Donde et al. [2001] has shown how LFC differs from standard implementation in case of the existence of bilateral contracts for energy trade among power market participants in different control areas. Their work considers only wholesale power market outcomes and not the reserve market outcomes. We proposed 

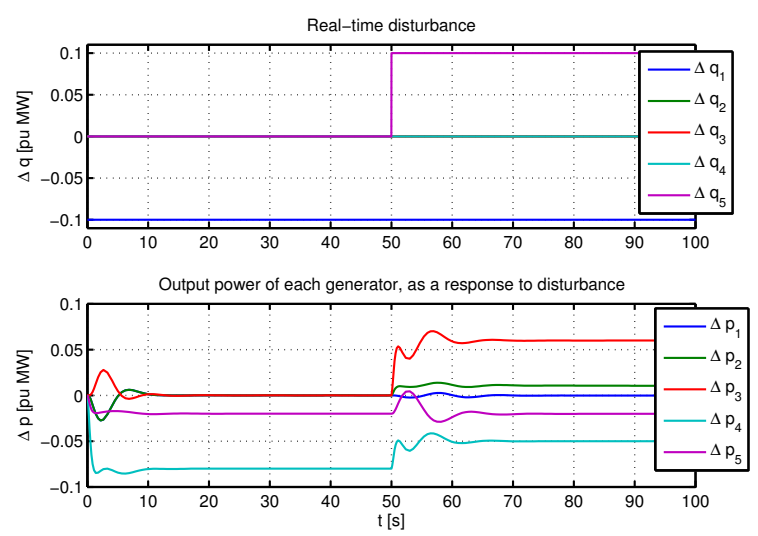

Fig. 6. Market outcome in case of cooperation among areas.
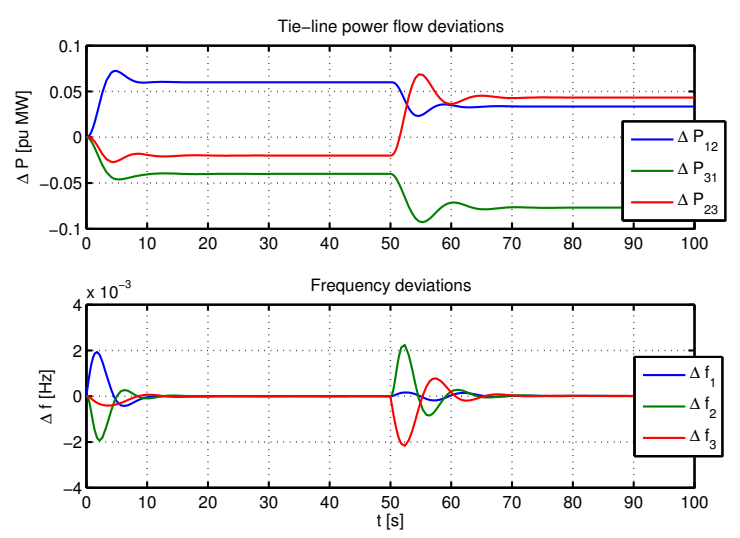

Fig. 7. Tie-line flow and frequency deviations in case of cooperation among areas.

the mapping that represents the balancing market outcome, no matter how the market is organized. The mapping covers the implementation of conventional markets for balancing reserves, the implementation of two-sided reserve market outcomes, and also the cooperation in reserve provision among different control areas. The characteristics of the mapping $g(\cdot)$ are discussed for every implementation. Our framework is in general agreement with the currently existing implementation. Hence, it is possible to have the proposed framework implemented in one control area only, while the others just keep the standard LFC implementation.

\section{REFERENCES}

R.J. Abraham, D. Das, and A. Patra. Bilateral load following in a restructured power system with local controllers. In Asian Control Conference, 2009. ASCC, pages 1199-1204, 2009.

V. Donde, M.A. Pai, and I.A. Hiskens. Simulation and optimization in an AGC system after deregulation. IEEE Transactions on Power Systems, 16(3):481-489, 2001.

A. Jokić. Price-based optimal control of electrical power systems. $\mathrm{PhD}$ thesis, 2007.

J. Kumar, K.H. Ng, and G. Sheble. AGC simulator for pricebased operation. Part I and Part II. IEEE Transactions on Power Systems, 12(2):527-538, 1997.

P. Kumar and D.P. Kothari. Recent philosophies of automatic generation control strategies in power systems. Power Systems, IEEE Transactions on, 20(1):346-357, 2005.
P. Kundur. Power System Stability and Control. McGraw-Hill Professional, 1994. ISBN 007035958X.

E. Nobile, A. Bose, and K. Tomsovic. Feasibility of a bilateral market for load following. IEEE Transactions on Power Systems, 16(4):782-787, 2001.

S. Stoft. Power System Economics: Designing Markets for Electricity. Wiley-IEEE Press, 2002. ISBN 0471150401.

TenneT. Congestion management, November 2011. Last accesed: November 2011.

Tractebel. Study of the interactions and dependencies of balancing markets, intraday trade and automatically activated reserves. Technical report, 2009. URL http://ec.europa.eu/energy/gas electricit y/studies/doc/electricity/2009 balancing. markets.pdf.

P.P.J. van den Bosch, A. Jokić, R.M. Hermans, J. Frunt, W.L. Kling, F. Nobel, P. Boonekamp, and W. de Boer. Reduced risks and improved economic operation of ancillary services. In 2010 7th International Conference on the European Energy Market. IEEE, 2010.

\section{APPENDIX}

For the completeness of the presentation, and in particular of proof of Proposition 5.2, here we prove the following wellknown characteristic of load frequency control (LFC).

Proposition 9.1. If area control error (ACE) in each control area with implemented LFC control is zero, then both, the frequency deviation $\Delta f_{i}$ of that area and the sum of all tie-line power flow deviations, $\Delta P_{A i}$ are zero.

Proof. ACE of a control area is defined as $A C E_{i}=\beta_{i} \Delta f_{i}+$ $\Delta P_{A i}$, where $\beta_{i}>0$, see Kundur [1994]. $\Delta P_{A i}$ represents the sum of all inter control-area tie-line power flows, $\Delta P_{i j}$. If $\Delta P_{i j}>0$, the excess power flows from node $n_{i}$ to node $n_{j}$.

Due to integral action, see Fig. 1, ACE is zero in steady state. In steady state $\beta_{i} \Delta f_{i}+\Delta P_{A i}=0, \forall i$ and $\Delta f_{i}=\Delta f_{j}$ for all pairs $(i, j)$. These equations can be stacked in a matrix form, with $K$ being the number of control areas

$$
\left[\begin{array}{cccccccc}
\beta_{1} & 0 & \ldots & 0 & 1 & 0 & \ldots & 0 \\
0 & \beta_{2} & \ldots & 0 & 0 & 1 & \ldots & 0 \\
\vdots & \vdots & \ddots & \vdots & \vdots & \vdots & \ddots & \vdots \\
0 & 0 & \ldots & \beta_{K} & 0 & 0 & \ldots & 1
\end{array}\right]\left[\begin{array}{c}
\Delta f_{1} \\
\vdots \\
\Delta f_{K} \\
\Delta P_{A 1} \\
\vdots \\
\Delta P_{A K}
\end{array}\right]=0_{K}
$$

As already mentioned, in steady state all $\Delta f_{i}$ are of the same value and the same sign, and $\beta_{i} \in \mathbb{R}_{+}$are positive parameters. Therefore, for equation (5) to hold, all $\Delta P_{A i}$ have to be of the same sign, which is opposite of the sign of frequency deviations.

Every power flow $\Delta P_{i j}$ is, on the level of overall system, taken into account twice: once while regulating $A C E_{i}$ and the second time while regulating $A C E_{j}$. If we now sum all $\Delta P_{A i}$ values, due to the fact the every flow is then taken into account twice, once with positive sign and other time with negative, we get $\sum_{i=1}^{K} \Delta P_{A i}=0$. Therefore, it is impossible for all $\Delta P_{A i}$ to be of the same sign. Therefore, $\Delta P_{A i}=0$ and $\Delta f_{i}=0$. 